\title{
Review Article \\ Review on the Research for Separated Continuous Linear Programming: With Applications on Service Operations
}

\author{
Xiaoqing Wang \\ Lingnan (University) College, Sun Yat-Sen University, Guangzhou, Guangdong 510275, China \\ Correspondence should be addressed to Xiaoqing Wang; xqwang1@gmail.com
}

Received 22 April 2013; Accepted 17 June 2013

Academic Editor: Tsan-Ming Choi

Copyright (C) 2013 Xiaoqing Wang. This is an open access article distributed under the Creative Commons Attribution License, which permits unrestricted use, distribution, and reproduction in any medium, provided the original work is properly cited.

We give a review on the research for a class of optimization model-separated continuous linear programming (SCLP). SCLP takes several similar forms and can be used to find the dynamic control of a multiclass fluid network. We review the duality theory and solution methods for it. We also present application examples of SCLP on service operations.

\section{Introduction}

Linear programming (LP) is probably the most successful mathematical model in terms of its extremely wide range of industrial applications and its superb speed and capacity in solving very large size problems. For this reason, LP has been pushed and extended to an ever-broadening frontier $[1,2]$. One of such frontier extensions is the so-called separated continuous linear programming(SCLP).

SCLP was first introduced by Anderson [3] who used it to model the job-shop scheduling problem. The following is the formulation according to him [3]:

$$
\begin{array}{ll}
\min & \int_{0}^{T} c(t)^{\prime} u(t) d t \\
\text { s.t. } & \int_{0}^{t} G u(s) d s \leq a(t), \\
& H u(t) \leq b(t), \\
& u(t) \geq 0, \quad t \in[0, T],
\end{array}
$$

where $u(t)$ is the decision variable and is assumed to be a bounded measurable function, $b(t), c(t)$ are bounded measurable functions, and $a(t)$ is an absolutely continuous function.
$G, F, H$ are constant matrices. “'” denotes the transpose operation. The word "separated" here refers to the fact that there are two kinds of constraints in SCLP: the constraints involving integration and the instantaneous constraints [3].

There are several other similar formulations of SCLP.

Luo and Bertsimas [4] considered the following more general SCLP which they called separated continuous linear programs with side constraints (SCSCLP):

$$
\begin{array}{ll}
\min & \int_{0}^{T}\left(c(t)^{\prime} u(t)+g(t)^{\prime} x(t)\right) d t \\
\text { s.t. } & \int_{0}^{t} G u(s) d s+E x(t) \leq a(t), \\
& H u(t) \leq b(t), \\
& F x(t) \leq h(t), \\
& u(t) \geq 0, \quad t \in[0, T],
\end{array}
$$

where $u(t), x(t)$ are decision variables and are assumed to be bounded measurable functions, $b(t), c(t), g(t)$, and $h(t)$ are bounded measurable functions, and $a(t)$ is an absolutely continuous function. It is easy to see that when $E, F$ are zero matrix, and $g(t)=0$ for $t \in[0, T],(2)$ is reduced to (1). 
Shapiro [5] considered the following SCLP problem:

$$
\begin{array}{ll}
\min & \int_{0}^{T} c(t)^{\prime} u(t) d t \\
\text { s.t. } & \int_{0}^{t} G(s, t) u(s) d s \leq a(t), \quad t \in[0, T], \\
& H(t) u(t) \leq b(t), \text { a.e. } t \in[0, T], \\
& u(t) \geq 0 \text {, a.e. } t \in[0, T],
\end{array}
$$

where $u(t)$ is the decision variable and is assumed to be a bounded measurable function, $b(t)$ and $H(t)$ are bounded measurable functions, $c(t)$ is a Lebesgue integrable function, $a(t)$ is a continuous function, and $G(s, t)$ is a continuous function such that, for every $s \in[0, T]$, it is of bounded variation as a function of $t \in[0, T]$. It is obvious that if $G(s, t)$ and $H(t)$ are constant matrices, (3) is reduced to (1). SCLP:

In the work of Weiss [6], he introduced the following

$$
\begin{array}{ll}
\max & \int_{0}^{T}\left((\gamma+(T-t) c)^{\prime} u(t)+d^{\prime} x(t)\right) d t \\
\text { s.t. } & \int_{0}^{t} G u(s) d s+F x(t) \leq \alpha+a t, \\
& H u(t) \leq b, \\
& u(t), x(t) \geq 0, \quad t \in[0, T],
\end{array}
$$

where $u(t), x(t)$ are decision variables and are assumed to be bounded measurable functions with the measure of the breakpoint set being 0 .

Wang [7] considered the following problem which she called generalized separated continuous linear programming (GSCLP):

$$
\begin{array}{ll}
\max & \int_{0}^{T}\left(c(T-t)^{\prime} u(t)+d(T-t)^{\prime} x(t)\right) d t \\
\text { s.t. } & a(t)-\int_{0}^{t} G u(s) d s-F x(t) \geq 0, \\
& b(t)-H u(t) \geq 0, \\
& u(t), x(t) \geq 0, \quad t \in[0, T]
\end{array}
$$

where $u(t), x(t)$ are decision variables and are assumed to be bounded measurable functions. $a(t), c(T-t)$ are piecewise linear, and $b(t), d(T-t)$ are piecewise constant function, on $[0, T]$. It is easy to see that when $a(t), c(t)$ are linear and $b(t)$, $d(t)$ are constant functions, (5) is reduced to (4).
In the recent paper by Nasrabadi et al. [8], they studied the SCLP with a fuzzy valued objective function which they called fuzzy separated continuous linear program (FSCLP):

$$
\begin{array}{cl}
\min & \int_{0}^{T}(\tilde{\gamma}+t \widetilde{c})^{\prime} u(t) d t \\
\text { s.t. } & \int_{0}^{t} G u(s) d s \leq \alpha+a t, \\
& H u(t) \leq b, \\
& u(t) \geq 0, \quad t \in[0, T] .
\end{array}
$$

Here, $u(t)$ is the decision variable and is assumed to be a bounded measurable function. $\widetilde{\gamma}, \widetilde{c}$ are constant vectors with trapezoidal fuzzy numbers as components.

The theoretical results for SCLP are mainly on the duality theory and solution methods. The application results for SCLP are on finding the dynamic control of a multiclass fluid network which can model alot of real systems, including service systems. In this paper, we review both the theoretical results and the application results for SCLP, with the focus of application on service operations.

The rest of the paper is organized as follows. In Section 2, we review the literature on duality theory for SCLP. Section 3 is devoted to the solution methods for SCLP. Application of SCLP on service operations is discussed in Section 4. We summarized the current results on SCLP and point out some future directions in this area in Section 5.

\section{Duality Theory}

As with finite LP, duality theory for SCLP plays an important role in the development of solution methods for it. In the following, we will discuss the results on duality theory for SCLP according to the formulation of SCLP.

Using essentially the same method as LP's, Anderson and Nash [9] derived a dual problem of SCLP (1) as in the following:

$$
\begin{array}{cc}
\max & -\int_{0}^{T} a(t)^{\prime} p(t) d t-\int_{0}^{T} b(t)^{\prime} q(t) d t \\
\text { s.t. } & c(t)+\int_{t}^{T} G^{\prime} p(s) d s+H^{\prime} q(t) \geq 0, \\
& p(t) \geq 0, q(t) \geq 0, \quad t \in[0, T],
\end{array}
$$

where $p(t), q(t)$ are the decision variables and are assumed to be bounded measurable functions. But later on, some researcher found that for (1) and (D1), there exist such instances that either the primal or the dual problem has no feasible or no optimal solution, while the other one has an optimal solution [10]. 
To avoid this difficulty, Pullan [11] introduced another dual problem of (1) as in the following:

$$
\begin{aligned}
& \max \quad-\int_{0}^{T} d \pi^{\prime}(t) a(t)-\int_{0}^{T} b(t)^{\prime} q(t) d t \\
& \text { s.t. } \quad c(t)-G^{\prime} \pi(t)+H^{\prime} q(t) \geq 0
\end{aligned}
$$

$\pi(t)$ monotonically increasing and right

continuous on $[0, T]$ with $\pi(T)=0$,

$q(t) \geq 0, \quad t \in[0, T]$,

where $\pi(t), q(t)$ are the decision variables and $q(t)$ is assumed to be a Lebesgue integrable function on $[0, T]$.

Pullan [12] developed an account of duality theory for (1) and $\left(D 1^{*}\right)$. He introduced the definition of complementary slackness for (1) and $\left(D 1^{*}\right)$ and proved that if $u(t)$ is feasible for (1) and $\left(\pi^{\prime}(t), q^{\prime}(t)\right)^{\prime}$ is feasible for $\left(D 1^{*}\right)$, when $\left(\pi^{\prime}(t), q^{\prime}(t)\right)^{\prime}$ complementary slack with $u(t), u(t)$ is optimal for (1) and $\left(\pi^{\prime}(t), q^{\prime}(t)\right)^{\prime}$ is optimal for $\left(D 1^{*}\right)$. He also derived several sufficient conditions for the optimality of (1), the conditions for no duality gap between (1) and $\left(D 1^{*}\right)$, and the conditions under which $\left(D 1^{*}\right)$ has an optimal solution. Finally, he proved that strong duality holds between (1) and $\left(D 1^{*}\right)$ and that there exist piecewise analytic optimal solutions for both (1) and $\left(D 1^{*}\right)$ under the assumptions that $a(t), b(t)$ and $c(t)$ are piecewise analytic and $a(t)$ is also continuous.

Luo and Bertsimas [4] formulated the dual problem of (2) as follows:

$$
\begin{aligned}
\max & -\int_{0}^{T} a(t)^{\prime} d \pi(t)-\int_{0}^{T} h(t)^{\prime} d p(t) \\
& -\int_{0}^{T} b(t)^{\prime} q(t) d t
\end{aligned}
$$

$$
\begin{array}{ll}
\text { s.t. } & c(t)-G^{\prime} \pi(t)+H^{\prime} q(t) \geq 0, \\
& E^{\prime} \pi(t)+F^{\prime} p(t)=\int_{t}^{T} g(t) d t
\end{array}
$$

$\pi(t)$ bounded measurable with

finite variation, $\pi(T)=0$,

$p(t)$ monotonically increasing and right

continuous on $[0, T]$ with $p(T)=0$,

$q(t) \geq 0, \quad t \in[0, T]$

where $\pi(t), p(t), q(t)$ are decision variables and $q(t)$ is assumed to be a bounded measurable function on $[0, T]$.
When $E, F$ are zero matrices and $g(t)=0$ for $t \in[0, T]$, $(D 2)$ is reduced to $\left(D 1^{*}\right)$.

They showed that there is no duality gap between (2) and (D2) under the following assumptions: $a(t)$ and $h(t)$ are continuous; $a(t), c(t)$ and $h(t)$ are piecewise linear; $b(t)$ and $g(t)$ are piecewise constant; (2) is feasible and its objective value is bounded from below.

Shapiro [5] derived the Lagrangian dual of (3) as follows:

$$
\begin{array}{ll}
\max & -\int_{0}^{T} a(t)^{\prime} d \pi(t)-\int_{0}^{T} b(t)^{\prime} q(t) d t \\
\text { s.t. } \quad & c(t)-G(t, t)^{\prime} \pi(t) \\
& -\int_{t}^{T}(d G(t, s))^{\prime} \pi(s)+H(t)^{\prime} q(t) \geq 0, \\
& q(t) \geq 0 \text {, a.e. } t \in[0, T], \\
& \pi(t) \text { monotonically nondecreasing, right } \\
& \text { continuous with bounded variation }
\end{array}
$$$$
\text { on }[0, T] \text { with } \pi(T)=0 \text {, }
$$

where $\pi(t), q(t)$ are decision variables and $q(t)$ is assumed to be a Lebesgue integrable function. When $G(s, t)$ and $H(t)$ are constant matrices, $(D 3)$ is reduced to $\left(D 1^{*}\right)$.

Shapiro proved that if the feasible region of (3) is nonempty, the matrix $H(t)$ is constant, and the set

$$
\{u(t): H u(t) \leq b(t), u(t) \geq 0 \text {, a.e. } t \in[0, T]\}
$$

is bounded, then the optimal objective value of (3) and (D3) are the same, and there exists an optimal solution for (3).

Weiss [6] gave the following dual of (4):

$$
\begin{array}{ll}
\min & \int_{0}^{T}\left((\alpha+(T-t) a)^{\prime} p(t)+b^{\prime} q(t)\right) d t \\
\text { s.t. } & \int_{0}^{t} G^{\prime} p(s) d s+H^{\prime} q(t) \geq \gamma+c t, \\
& F^{\prime} p(t) \geq d, \\
& p(t), q(t) \geq 0, \quad t \in[0, T],
\end{array}
$$

where $p(t), q(t)$ are decision variables and are assumed to be bounded measurable functions. Note that in this dual problem, the time is running back, that is, here $t$ is $T-t$ in the primal problem.

Weiss introduced the following Boundary-LP, Boundary$\mathrm{LP}^{*}$, Rates-LP $(K, J)$, and Rates-LP* $(K, J)$. 
Boundary-LP

$$
\begin{array}{cc}
\max & d^{\prime} x^{0} \\
\text { s.t. } & F x^{0} \leq \alpha, \\
& x^{0} \geq 0,
\end{array}
$$

Boundary-LP*

$$
\begin{array}{ll}
\min & b^{\prime} q^{N} \\
\text { s.t. } & H^{\prime} q^{N} \geq \gamma, \\
& q^{N} \geq 0,
\end{array}
$$

Rates-LP $(K, J)$

$$
\begin{array}{ll}
\max & {\left[\begin{array}{ll}
c^{\prime} & 0
\end{array}\right] u+\left[\begin{array}{ll}
0 & d^{\prime}
\end{array}\right] \dot{x}} \\
\text { s.t. } & {\left[\begin{array}{ll}
G & 0
\end{array}\right] u+\left[\begin{array}{ll}
I & F
\end{array}\right] \dot{x}=a,} \\
& {\left[\begin{array}{ll}
H & I
\end{array}\right] u=b,} \\
& \dot{x}_{k} \geq 0 \text { for } k \notin K, \\
& u_{j}=0 \text { for } j \in J, \\
& u_{j} \geq 0 \text { for } j \notin J,
\end{array}
$$

Rates-LP* $(K, J)$

$$
\begin{array}{ll}
\min & {\left[\begin{array}{ll}
a^{\prime} & 0
\end{array}\right] p+\left[\begin{array}{ll}
0 & b^{\prime}
\end{array}\right] \dot{q}} \\
\text { s.t. } & {\left[\begin{array}{ll}
G^{\prime} & 0
\end{array}\right] p+\left[\begin{array}{ll}
-I & H^{\prime}
\end{array}\right] \dot{q}=c,} \\
& {\left[\begin{array}{ll}
F^{\prime} & -I
\end{array}\right] p=d,} \\
& \dot{q}_{j} \geq 0 \quad \text { for } j \notin J, \\
& p_{k}=0 \quad \text { for } k \in K, \\
& p_{k} \geq 0 \quad \text { for } k \notin K,
\end{array}
$$

where $K, J$ are the subsets of the subscripts for $x(t)(p(t)), u(t)(q(t))$ in (4), respectively. Weiss proved that strong duality holds between (4) and (D4) under Assumption 1.

\section{Assumption 1.}

(i) The Boundary-LP/LP* have a solution $x^{0}, q^{N}$. Denote $K_{0}=\left\{k: x_{k}^{0}>0\right\}, J_{N}=\left\{j: q_{j}^{N}>0\right\}$.

(ii) The Rates-LP $\left(\emptyset, J_{N}\right)$ and the Rates-LP* $\left(K_{0}, \emptyset\right)$ are both feasible.
Wang [7] considered the following dual of (5):

$$
\begin{array}{ll}
\min & \int_{0}^{T}\left(a(T-t)^{\prime} p(t)+b(T-t)^{\prime} q(t)\right) d t \\
\text { s.t. } & \int_{0}^{t} G^{\prime} p(s) d s+H^{\prime} q(t)-c(t) \geq 0, \\
& F^{\prime} p(t)-d(t) \geq 0, \\
& p(t), q(t) \geq 0, \quad t \in[0, T],
\end{array}
$$

where $p(t), q(t)$ are decision variables and are assumed to be bounded measurable functions. When $a(t), c(t)$ are linear $b(t), d(t)$ are constant functions, (D5) is reduced to (D4).

Wang [7] proved that strong duality holds between (5) and (D5) under Assumption 2.

Assumption 2. The following two ordinary linear programming problems have optimal solutions:

$$
\begin{aligned}
& \min \left(\begin{array}{c}
\left(T-T_{1}\right) a_{2} \\
T_{1} a_{1}
\end{array}\right)^{\prime} p+\left(\begin{array}{c}
\left(T-T_{1}\right) b_{2}-T_{1} b_{1} \\
T_{1} b_{1}
\end{array}\right)^{\prime} q \\
& \text { s.t. } \quad\left(\begin{array}{ll}
G^{\prime} & \\
G^{\prime} & G^{\prime}
\end{array}\right) p+\left(\begin{array}{ll}
H^{\prime} & \\
& H^{\prime}
\end{array}\right) q \\
& -\left(\begin{array}{c}
\gamma+\left(T-T_{1}\right) c_{1} \\
\gamma+\left(T-T_{1}\right) c_{1}+T_{1} c_{2}
\end{array}\right) \geq 0, \\
& \left(\begin{array}{cc}
F^{\prime} & \\
& F^{\prime}
\end{array}\right) p-\left(\begin{array}{c}
\left(T-T_{1}\right) d_{1} \\
T_{1} d_{2}
\end{array}\right) \geq 0, \\
& p, q \geq 0 \text {, } \\
& \max \left(\begin{array}{c}
\left(T-T_{1}\right) c_{1} \\
T_{1} c_{2}
\end{array}\right)^{\prime} u+\left(\begin{array}{c}
\left(T-T_{1}\right) d_{1} \\
T_{1} d_{2}-\left(T-T_{1}\right) d_{1}
\end{array}\right)^{\prime} x \\
& \text { s.t. } \quad\left(\begin{array}{c}
\alpha+T_{1} a_{1}+\left(T-T_{1}\right) a_{2} \\
\alpha+T_{1} a_{1}
\end{array}\right) \\
& -\left(\begin{array}{cc}
G & G \\
& G
\end{array}\right) u-\left(\begin{array}{ll}
F & \\
& F
\end{array}\right) x \geq 0, \\
& \left(\begin{array}{c}
\left(T-T_{1}\right) b_{2} \\
T_{1} b_{1}
\end{array}\right)-\left(\begin{array}{cc}
H & \\
& H
\end{array}\right) u \geq 0,
\end{aligned}
$$

$u, x \geq 0$.

Nasrabadi et al. [8] consider the following dual problem of (6):

$$
\begin{aligned}
& \max \quad-\int_{0}^{T}(\alpha+t a) d \tilde{\pi}(t)^{\prime}-\int_{0}^{T} b^{\prime} \tilde{q}(t) d t \\
& \text { s.t. } \quad \tilde{\gamma}+t \tilde{c}-G^{\prime} \tilde{\pi}(t)+H^{\prime} \tilde{q}(t) \geq_{R} 0,
\end{aligned}
$$

$\tilde{\pi}(t)$ is trapezoidal fuzzy number-valued, monotonically increasing, and right continuous 
on $[0, T]$ with $\tilde{\pi}(T)=0$,

$\tilde{q}(t)$ is Lebesgue-integrable and

trapezoidal fuzzy numbervalued,

where $\tilde{\pi}(t), \tilde{q}(t)$ are decision variables and $R$ denotes any arbitrary, but fixed, linear-ranking function.

They proved that strong duality holds between (6) and (D6) with respect to $R$ under the assumption that (6) has an optimal solution.

\section{Solution Methods}

One of the motivations for studying SCLP is that under some assumptions, the solution for SCLP has some nice forms. In the following, we introduce the results in the form of the optimal solution for SCLP under various assumptions in the first subsection; we present the algorithms for solving SCLP problem in the second to fourth subsections.

3.1. The Form of the Optimal Solution for SCLP. Anderson et al. [13] pointed out that if the feasible region for (1) is nonempty and bounded, then (1) is solvable and there exists an extreme point optimal solution for (1). Furthermore, if $a(t), c(t)$ are piecewise linear, with $a(t)$ also absolutely continuous, and $b(t)$ is piecewise constant, then (1) has an optimal solution in which $u(t)$ is piecewise constant on $[0, T]$.

Anderson and Philpott [14] continued to study this issue and proved that when $a(t)$ and $b(t)$ are piecewise analytic (but with $a(t)$ continuous) and $c(t)$ is piecewise constant on $[0, T]$, if the set $\{u(t): H u(t) \leq b(t), u(t) \geq 0\}$ is bounded for each $t \in[0, T]$ and the feasible region for (1) is nonempty, then there exists an optimal solution for (1) with $u(t)$ piecewise analytic on $[0, T]$.

Pullan [15] proved that if $a(t), b(t), c(t)$ are piecewise analytic on $[0, T]$, with $a(t)$ also continuous, when the feasible region for (1) is nonempty and bounded, there exists an optimal extreme point solution for (1) with $u(t)$ piecewise analytic on $[0, T]$. Furthermore, if $a(t)$ is piecewise linear and $b(t)$ is piecewise constant on $[0, T]$, there exists an optimal extreme point solution for (1) with $u(t)$ piecewise constant on $[0, T]$.

Note that the results of $[11,12]$ do not guarantee an optimal solution of the appropriate form that is also an extreme point; the results in [15] do guarantee such a solution.

Luo and Bertsimas [4] showed that there exists an optimal solution for $(D 2)$ that is piecewise linear under the following assumption: $a(t)$ and $h(t)$ are continuous; $a(t), c(t)$ and $h(t)$ are piecewise linear; $b(t)$ and $g(t)$ are piecewise constant; (2) is feasible and its objective value is bounded from below. Furthermore, there exists a bounded measurable optimal solution for (2) if and only if the algorithm they suggested terminates with such a solution. Also, when the feasible region for (2) is bounded and $E$ is an identity matrix, there exists an optimal solution in which $u(t)$ is piecewise constant.
Weiss [6] proved that under Assumption 1, (4) and (D4) possess complementary slack optimal primal and dual solutions, with continuous piecewise linear $x(t), q(t)$ and piecewise constant $u(t), p(t)$.

Wang [7] proved that under Assumption 2, there exist optimal solutions for (5) in which $u(t)$ is piecewise constant and $x(t)$ is piecewise linear, or there exist a series of feasible solutions for (5) in which $u(t)$ is piecewise constant and $x(t)$ is piecewise linear and whose objective values converge to the optimal objective value of (5).

Nasrabadi et al. [8] proved that under the assumption that the feasible region for (6) is bounded and nonempty, there exist optimal solutions for $(6)$ in which $u(t)$ is piecewise constant and $x(t)$ is piecewise linear, or there exist a series of feasible solutions for (6) in which $u(t)$ is piecewise constant and $x(t)$ is piecewise linear and whose objective values converge to the optimal objective value of (6).

3.2. Simplex-Like Methods for Solving SCLP Problem. Based on the duality theory and results on the forms of the optimal solutions for SCLP, several methods are proposed to solve SCLP exactly or approximately. Most of these algorithms fall in one of two categories: simplex-like and discretization based. In this subsection, we will discuss the simplex-like methods. The discretization methods will be discussed in the next subsection.

Anderson and Philpott [16] developed the so-called continuous-time network simplex algorithm to solve a kind of continuous network program which can be formulated as (1) with piecewise linear $a(t)$ and $c(t)$, with $a(t)$ being absolutely continuous, and piecewise constant $b(t)$. This is the first algorithm which was implemented in a computer to solve (1). Unfortunately, there is no convergence guarantee for this algorithm, and it often produces a sequence of solutions which converge to a suboptimal solution [17].

Pullan [18] proposed a simplex-like algorithm to solve (1) with piecewise linear and continuous $a(t)$, piecewise constant $b(t)$, and piecewise analytic $c(t)$. Again, there is no convergence guarantee for this algorithm.

In the recent work of Weiss [6], he proposed a simplexlike algorithm to solve (4) under Assumptions 1 and 3 as follows.

Assumption 3. The column $\left[\begin{array}{l}a \\ b\end{array}\right]$ is in general position to the matrix $\left[\begin{array}{cccc}G & F & I & 0 \\ H & 0 & 0 & I\end{array}\right]$, and the column $\left[\begin{array}{l}c \\ d\end{array}\right]$ is in general position to the matrix $\left[\begin{array}{cccc}G^{\prime} & H^{\prime} & -I & 0 \\ F^{\prime} & 0 & 0 & -I\end{array}\right]$.

The Weiss algorithm is a parametric and recursive algorithm: start from an optimal solution valid at $T=0$ and construct the optimal solution valid at $T \in\left[\theta^{r-1} T, \theta^{r} T\right]$, where $r=1, \ldots, R$ and $0=\theta^{0}<\theta^{1}<\cdots<\theta^{R}=1$, and finally, reach the optimal solution at $T$. Going from optimal solution valid at $T \in\left[\theta^{r-1} T, \theta^{r} T\right]$ to that valid at $T \in\left[\theta^{r} T, \theta^{r+1} T\right]$ constitutes the pivot operation of SCLP, which involves solving several Rates-LP and Rates-LP* and/or solving several subproblems of original SCLP (4) which are themselves SCLP's with smaller size than that of (4).

Weiss managed to prove that the number of SCLP pivots needed to solve (4) is finite but increase exponentially with the problem size. 
3.3. Discretization-Based Methods for Solving SCLP Problem. There are two kinds of discretization-based methods, one is solving a sequence of discretization problems for SCLP and terminated with some predefined criteria; the other is solving a specific instance of discretization problems for SCLP and terminated with the solution with some pre-defined precision.

Pullan [19] proposed an algorithm to solve (1) with piecewise linear $a(t), c(t)$, with $a(t)$ also absolutely continuous, and piecewise constant $b(t)$. He formulated a new discretization for (1) which is a finite LP problem (there is another discretization problem for (1) called the standard discretization for (1) which is also a finite LP problem. The later was used before in the context of CLP in, e.g., [20, 21]). Based on the relationship between (1), $\left(D 1^{*}\right)$, and the discretization problems he introduced, a feasible solution for (1) is obtained with $u(t)$ piecewise constant in $[0, T]$. Then, in each iteration, the existing feasible solution for (1) is used together with the solution for the discretization he introduced to produce a new feasible solution with the strictly improving objective value. The number of the breakpoints in the new feasible solution is tripled. This process continues until an optimal solution is found or the resulting feasible solution is within a predescribed limit. The algorithm either terminates in a finite number of iterations with an optimal solution, or the objective values of the resulting feasible iterative solutions converge to the optimal objective value of (1).

Following Pullan's work, Philpott and Craddock [17] proposed a similar algorithm for (1). The main difference between their algorithm and Pullan's is in the criterion for adding breakpoints in producing the new feasible solution. After each iteration, the number of breakpoints in the new feasible solution Philpott and Craddock [17] produced is at most doubled.

Luo and Bertsimas [4] provided an algorithm to solve (2) under the assumptions that $a(t)$ and $h(t)$ are continuous; $a(t)$, $c(t)$ and $h(t)$ are piecewise linear; $b(t)$ and $g(t)$ are piecewise constant; (2) is feasible and its objective value is bounded from below. The main idea of their algorithm is similar to that of Pullan [19] and Philpott and Craddock [17] for (1). The key difference is that in Pullan [19] and Philpott and Craddock [17], at each iteration, the breakpoints in $[0, T]$ are fixed so that the discretization problem for (1) is an LP problem; In this algorithm, the breakpoints of $[0, T]$ are also a decision variable (although the number of the breakpoints is fixed), so the discretization problem for (2) is a (in general) nonconvex quadratic programming problem. In each iteration, this nonconvex quadratic programming problem is solved to get a KKT solution. The series of KKT points obtained have nonincreasing objective value. These KKT solutions are used to construct a sequence of feasible solutions for (2) with piecewise constant $u(t)$, and the objective values of these feasible solutions converge to the optimal objective value of (2). If the feasible region for (2) is bounded and $E$ is the identity matrix, the algorithm produces an optimal solution for (2) with piecewise constant $u(t)$.

Nasrabadi et al. [8] proposed an algorithm to solve (6) under the assumption that the feasible region for (6) is bounded and nonempty. Their algorithm is the counterpart of Pullan's algorithm [19] for solving (6). After each iteration, their algorithm constructs a new feasible solution for (6) with the number of breakpoints doubled.

All the discretization-based algorithms we have discussed so far solve a sequence of discretization problems for SCLP until the optimal solution is obtained or some pre-defined criteria are met. However, the following two algorithms only solve a specific instance of the discretization problem for SCLP and get a feasible solution with pre-defined quality.

The first of these algorithms is proposed by Fleischer and Sethuraman [22]. Their algorithm is used to solve a special case of multicommodity flow problem with holding costs (MHC) which can be written as (2) with $x(T)=0$, and $a(t)$, $b(t), c(t), g(t), h(t)$ are nonnegative constant vectors, and $E$ is the identity matrix, with the assumption that the objective value of this (2) is bounded below by 0 . It is not known if there is any optimal solution for this problem because the solution set of this problem may not be bounded. By analyzing the properties of the objective function, they introduced a discretization problem (which is an LP problem) with two given constants $\varepsilon>0$ and $\sigma>0$. Solving this discretization problem is essentially finding a minimum cost flow in the timeexpanded network of the original network. This algorithm will produce a feasible solution with the objective value $(1+$ $\varepsilon)$ opt $+\delta$, where opt is the optimal objective value of the original problem. The time complexity of this algorithm is polynomial in the input network, $1 / \varepsilon$ and $\log (1 / \delta)>0$.

The second of these algorithms is proposed by Wang [7]. The pre-defined error bound between the objective value of the required solution and the optimal objective solution for (5) is denoted as $\delta$. By analyzing the relationship between the discretizations for (5) and $\delta$, one specific discretization problem for (5) is solved and the solution obtained is used to construct a feasible solution for (5) with piecewise constant $u(t)$ and piecewise linear $x(t)$. The difference between the objective value of this feasible solution and the optimal objective solution for (5) is bounded by $\delta$.

3.4. Other Methods for Solving the SCLP Problem. There are still some solution methods for solving SCLP which do not fall in the previous two categories.

Chen and Yao [23] developed a myopic approach to solve the SCLP by solving a sequence of LP's over time. They gave sufficient conditions under which the myopic approach results in an optimal solution. They also showed that for the so-called Klimov's model-a multiclass network with a single server-this approach leads to the same priority policy as in Klimov's original stochastic setting, which is known as the Gittins index rule.

Avram et al. [24] solve (1) with linear $a(t), c(t)$ and constant $b(t)$ by heuristic method. By using Pontryagin's maximum principle, they show that the optimal solution is the threshold type control. They proposed an algorithm that first decomposes the original problem into several very small size problems and uses maximum principle to derive the exact optimal solutions for these problems; the solutions obtained are then combined to construct an approximate solution for the original problems. 


\section{Application on Service Operations}

SCLP has in recent years attracted considerable research attention in the field of stochastic networks. The multiclass stochastic network is a system that consists of different classes (types) of jobs which need to be processed and a set of servers which process the jobs. Jobs arrive to the system randomly or according to some probability distribution. Each server can process one or more classes of jobs, and the processing time for each server to process one job is different for different classes of jobs. The jobs in the same class have the same characteristics such as arrival rate and service requirements After one job is processed in one server, it may leave the network instantaneously or may become another class of jobs and go to another server for processing. The multiclass stochastic network is a very useful model for many real systems. For example, it can model the supply chain in which different products are delivered [25]. It can model the hub-and-spoke network in airline systems with crowdedness or congestion in the system [26]. It also can model the operations of call centers with interactive voice response [27]. It can also model health units with many patients in them [28].

For each multiclass stochastic network, there is a corresponding deterministic fluid network, which takes only the first-order data (means and rates) from the stochastic model and assumes that the jobs circulating in the network are continuous flows instead of discrete units. With appropriate scaling, the fluid network is a limit of the stochastic network, in the sense of strong law of large numbers (refer to, e.g., [29]). Furthermore, the fluid model has played a central role in studying the stability of stochastic networks [30]. Because of these developments, the real-time control (dynamic scheduling) of a stochastic network, which is itself a quite intractable problem, can be turned into the control of a corresponding fluid network, and the latter problem takes exactly the form of SCLP.

Although the most applications of SCLP mentioned in the literature are in the manufacturing system, they do have some applications in service systems which we will present in the next two subsections. Note that these applications are for the corresponding fluid networks instead of the original networks.

4.1. Optimal Dynamic Routing (Evacuation) in Communication Networks. Hajek and Ogier [31] considered a single destination communication network $\Phi=(V, A, c, d)$, where $(V, A)$ is a finite directed graph with a set $V$ of nodes and a set $A$ of links. $A \subset V \times V, d$ is the destination node, and $c=\left(c_{l}: l \in A\right)$ is a capacity assignment vector, $c_{l} \geq 0$ for every link $l$.

For each node $i$ in $V$, let $x_{i}(t)$ denote the amount of traffic at node $i$ at time $t$. A demand for the network is a pair $(x(0), r)$ where $x_{i}(0)$ denotes the (non-negative) initial amount of traffic at node $i$ and $r_{i}$ denotes the (non-negative) rate of traffic arriving at node $i$ from outside the network. By convention, $x_{d}(t)=r_{d}=0$ for all $t \geq 0$. Since the input rates $r_{i}$ are not time varying, if the total delay is finite for some control, then it is possible to empty each of the nodes in finite time. Let $T$ denote such a time point that $x(T)=0$. Let $u_{l}(t)$ denote the instantaneous flow on link $l$ at time $t$; the problem is finding a measurable function $u(t)$, such that the total waiting time in the network incurred by all traffic is minimized.

For every $i \in V$, let $E(i)$ denote the collection of nodes $j$ such that $(i, j) \in A$; let $I(i)$ denote the collection of nodes $k$ such that $(k, i) \in A$.

The problem can be formulated as follows:

$$
\begin{array}{ll}
\min & \int_{0}^{T} \sum_{i \in N-d} x_{i}(t) d t \\
\text { s.t. } & \dot{x}_{i}(t)=r_{i}-\sum_{j \in E(i)} u_{i j}(t) \\
& +\sum_{k \in I(i)} u_{k i}(t), \quad i \in V, \\
& u_{l}(t) \leq c_{l}, \quad l \in A, \\
& u_{l}(t) \geq 0, \quad x_{i}(t) \geq 0 .
\end{array}
$$

By integration on both sides of the first constraint from $[0, T]$, it is easy to see that this is a special case of SCLP (4).

4.2. Admission Control and Dynamic Routing for the Telephone Loss Network. Luo [32] considered a telephone loss network defined on a complete digraph $G=(V, A)$, with $V=$ $\{1,2, \ldots, n\}$ and $A=\{(i, j), i \neq j\}$, that is, this network consists of $n$ different locations $i=1, \ldots, n$ and $n \times(n-1)$ different links $(i, j)$, for $i \neq j$. At time 0 , there are some initial calls in the network. From each $i$, calls to $j$ arise at a rate of $\lambda_{i j}$, and the duration of each is $1 / \mu$. Calls will either be accepted or rejected. If a call from $i$ to $j$ is accepted, it generates reward $w_{i j}$ and can either be routed directly to $j$ through the link $(i, j)$ or be routed through $(i, k)$ to a third location $k$ and then from $k$ to $j$ through $(k, j)$. There are no other alternative routes, and once a call is accepted, it cannot be interrupted. Every link $(i, j)$ has a capacity of $c_{i j}$ switching circuits. Every call consumes one switching circuit on every link it uses. If a call from $i$ to $j$ is rejected, a penalty of $v_{i j}$ is incurred.

For any $i \neq j$ and $k \neq j$, let $x_{i k j}(t)$ be the number of calls at time $t$ in the network that are routed from $i$ to $j$ through $k$, and let $x_{i i j}(t)$ be the number of calls at time $t$ that are routed directly from $i$ to $j$. For any $i \neq j$ and $k \neq j$, let $u_{i k j}(t)$ be the control variable that represents the rate at which calls made at time $t$ from $i$ to $j$ through $k$, and let $u_{i i j}(t)$ be the rate at which calls made at time $t$ are routed directly from $i$ to $j$. The problem is to decide whether to accept a call, and if we accept it, how are we going to route it, so that the net revenue (the sum of the weighted rewards of accepted calls less the penalty for lost calls) is maximized over a period of time $[0, T]$. 
The problem can be formulated as follows:

$$
\begin{array}{ll}
\max & \int_{0}^{T}\left(\sum_{i, j} w_{i j} \sum_{k} x_{i k j}(t)\right. \\
& \left.-\sum_{i, j} v_{i j}\left(\lambda_{i j}-\sum_{k} u_{i k j}(t)\right)\right) d t \\
\text { s.t. } \quad & x_{i k j}(t)=x_{i k j}(0) \\
& +\int_{0}^{t}\left(u_{i k j}(t)-\mu x_{i k j}(t)\right) d t, \quad i \neq j, k \neq j \\
& \sum_{k \neq j} u_{i k j}(t) \leq \lambda_{i j}, \quad i \neq j \\
& \sum_{k \neq j} x_{k i j}(t)+\sum_{j \neq k, i \neq k} x_{i j k}(t) \leq c_{i j}, \quad i \neq j \\
& x(t), u(t) \geq 0, \quad t \in[0, T] .
\end{array}
$$

With a slight abuse of notation, define $x_{i k j}(t)=x_{i k j}(t) e^{\mu t}$ and $u_{i k j}(t)=u_{i k j}(t) e^{\mu t}$. This problem can be reformulated as follows:

$$
\begin{array}{cl}
\max & \int_{0}^{T}\left(e^{-\mu t} v^{\prime} u(t)+e^{-\mu t} w^{\prime} x(t)\right) d t \\
\text { s.t. } \quad & x_{i k j}(t)=x_{i k j}(0) \\
& +\int_{0}^{t} u_{i k j}(t) d t, \quad i \neq j, k \neq j \\
& \sum_{k \neq j} u_{i k j}(t) \leq e^{\mu t} \lambda_{i j}, \quad i \neq j \\
\sum_{k \neq j} x_{k i j}(t)+\sum_{j \neq k, i \neq k} x_{i j k}(t) \leq e^{\mu t} c_{i j}, \quad i \neq j \\
x(t), u(t) \geq 0, \quad t \in[0, T] .
\end{array}
$$

It is easy to see that this is an SCLP (2).

\section{Conclusion}

In this paper, we give a review on the research for SCLP. Several formulations of SCLP are presented, along with the related duality theory and solution method. Most results on duality theory provide the conditions under which SCLP has strong duality or has optimal solution. Most solution methods for SCLP fall in two categories: simplex-like and discretization based. The simplex-like method can get the exact optimal solution but is very time consuming; the discretization based methods are quite fast but can only get the approximate solutions in most cases.

There are still some problems needed to address in the future. For example, how to perform the sensitivity analysis for SCLP? How to define the robust SCLP? As the parameters in SCLP may not be able to get accurately in practice, the results of these research directions will definitely help to enlarge the range of the applications of SCLP.

\section{References}

[1] X. Ding and C. Wang, "A novel algorithm of stochastic chanceconstrained linear programming and its application," Mathematical Problems in Engineering, vol. 2012, Article ID 139271, 17 pages, 2012.

[2] W. Chen, Y. Yang, and H. Ma, "Fuzzy portfolio selection problem with different borrowing and lending rates," Mathematical Problems in Engineering, vol. 2011, Article ID 263240, 15 pages, 2011.

[3] E. J. Anderson, A continuous model for job-shop scheduling [Ph.D. thesis], University of Cambridge, Cambridge, UK, 1978.

[4] X. Luo and D. Bertsimas, "A new algorithm for state-constrained separated continuous linear programs," SIAM Journal on Control and Optimization, vol. 37, no. 1, pp. 177-210, 1998.

[5] A. Shapiro, "On duality theory of conic linear problems," in Semi-Infinite Programming, M. A. Goberna and M. A. Lopez, Eds., vol. 57, pp. 135-165, Kluwer Academic Publishers, Dordrecht, The Netherlands, 2001.

[6] G. Weiss, "A simplex based algorithm to solve separated continuous linear programs," Mathematical Programming, vol. 115, no. 1, pp. 151-198, 2008.

[7] X. Wang, "The duality theory of generalized separated continuous linear programming," in Proceedings of the International Conference on Management and Service Science (MASS '10), pp. 24-26, Wuhan, China, August 2010.

[8] M. M. Nasrabadi, M. A. Yaghoobi, and M. Mashinchi, "Separated continuous linear programs with fuzzy valued objective function," Scientia Iranica D, vol. 17, no. 2, pp. 105-118, 2010.

[9] E. J. Anderson and P. Nash, Linear Programming in InfiniteDimensional Spaces: Theory and Applications, Wiley-Interscience Series in Discrete Mathematics and Optimization, John Wiley \& Sons, Chichester, UK, 1987.

[10] R. C. Grinold, Continuous programming [Ph.D. thesis], Operations Research Center, University of Berkeley, Berkeley, Calif, USA, 1968.

[11] M. C. Pullan, "An algorithm for a class of continuous linear programs," SIAM Journal on Control and Optimization, vol. 31, no. 6, pp. 1558-1577, 1993.

[12] M. C. Pullan, "A duality theory for separated continuous linear programs," SIAM Journal on Control and Optimization, vol. 34, no. 3, pp. 931-965, 1996.

[13] E. J. Anderson, P. Nash, and A. F. Perold, "Some properties of a class of continuous linear programs," SIAM Journal on Control and Optimization, vol. 21, no. 5, pp. 758-765, 1983.

[14] E. J. Anderson and A. B. Philpott, "On the solutions of a class of continuous linear programs," SIAM Journal on Control and Optimization, vol. 32, no. 5, pp. 1289-1296, 1994.

[15] M. C. Pullan, "Forms of optimal solutions for separated continuous linear programs," SIAM Journal on Control and Optimization, vol. 33, no. 6, pp. 1952-1977, 1995.

[16] E. J. Anderson and A. B. Philpott, "A continuous-time network simplex algorithm," Networks, vol. 19, no. 4, pp. 395-425, 1989.

[17] A. B. Philpott and M. Craddock, "An adaptive discretization algorithm for a class of continuous network programs," Networks, vol. 26, no. 1, pp. 1-11, 1995.

[18] M. C. Pullan, "An extended algorithm for separated continuous linear programs," Mathematical Programming, vol. 93, no. 3, pp. 415-451, 2002.

[19] M. C. Pullan, "Convergence of a general class of algorithms for separated continuous linear programs," SIAM Journal on Optimization, vol. 10, no. 3, pp. 722-731, 2000. 
[20] R. N. Buie and J. Abrham, "Numerical solutions to continuous linear programming problems," Zeitschrift für Operations Research, vol. 17, no. 3, pp. 107-117, 1973.

[21] W. F. Tyndall, "A duality theorem for a class of continuous linear programming problems," Journal of the Society for Industrial and Applied Mathematics, vol. 13, no. 3, pp. 644-666, 1965.

[22] L. Fleischer and J. Sethuraman, "Efficient algorithms for separated continuous linear programs: the multicommodity flow problem with holding costs and extensions," Mathematics of Operations Research, vol. 30, no. 4, pp. 916-938, 2005.

[23] H. Chen and D. D. Yao, "Dynamic scheduling of a multiclass fluid network," Operations Research, vol. 41, no. 6, pp. 1104-1115, 1993.

[24] F. Avram, D. Bertsimas, and M. Ricard, "Fluid models of sequencing problems in open queueing networks; an optimal control approach," in Stochastic Networks, F. P. Kelly and R. Williams, Eds., vol. 71 of IMA Volumes in Mathematics and Its Applications, pp. 199-234, Springer, New York, NY, USA, 1995.

[25] V. Bhaskar and P. Lallement, "Modeling a supply chain using a network of queues," Applied Mathematical Modelling, vol. 34, no. 8, pp. 2074-2088, 2010.

[26] M. Mohammadi, F. Jolai, and H. Rostami, "An M/M/c queue model for hub covering location problem," Mathematical and Computer Modelling, vol. 54, no. 11-12, pp. 2623-2638, 2011.

[27] C. Kim, A. Dudin, S. Dudin, and O. Dudina, “Tandem queueing system with impatient customers as a model of call center with interactive voice response," Performance Evaluation, vol. 70, no. 6, pp. 440-453, 2013.

[28] M. Asaduzzaman, T. J. Chaussalet, and N. J. Robertson, "A loss network model with overflow for capacity planning of a neonatal unit," Annals of Operations Research, vol. 178, no. 1, pp. 67-76, 2010.

[29] H. Chen and D. D. Yao, Fundamentals of Queueing Networks: Performance, Asymptotics, and Optimization, vol. 46 of Applications of Mathematics, Springer, New York, NY, USA, 2001.

[30] J. G. Dai, "On positive Harris recurrence of multiclass queueing networks: a unified approach via fluid limit models," The Annals of Applied Probability, vol. 5, no. 1, pp. 49-77, 1995.

[31] B. Hajek and R. G. Ogier, "Optimal dynamic routing in communication networks with continuous traffic," Networks, vol. 14 , no. 3, pp. 457-487, 1984.

[32] X. Luo, Continuous linear programming: theory, algorithms and applications [Ph.D. thesis], Massachusetts Institute of Technology, Cambridge, Mass, USA, 1995. 


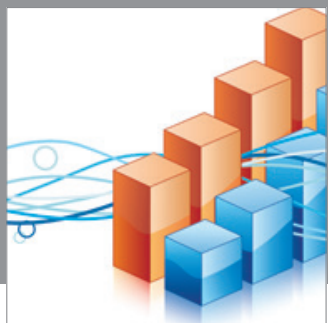

Advances in

Operations Research

mansans

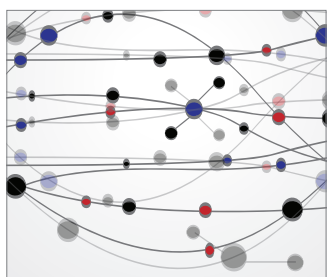

The Scientific World Journal
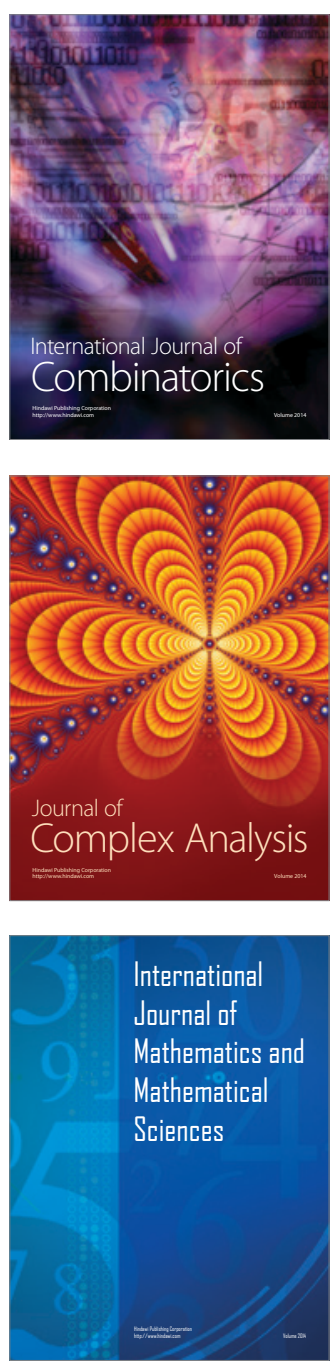
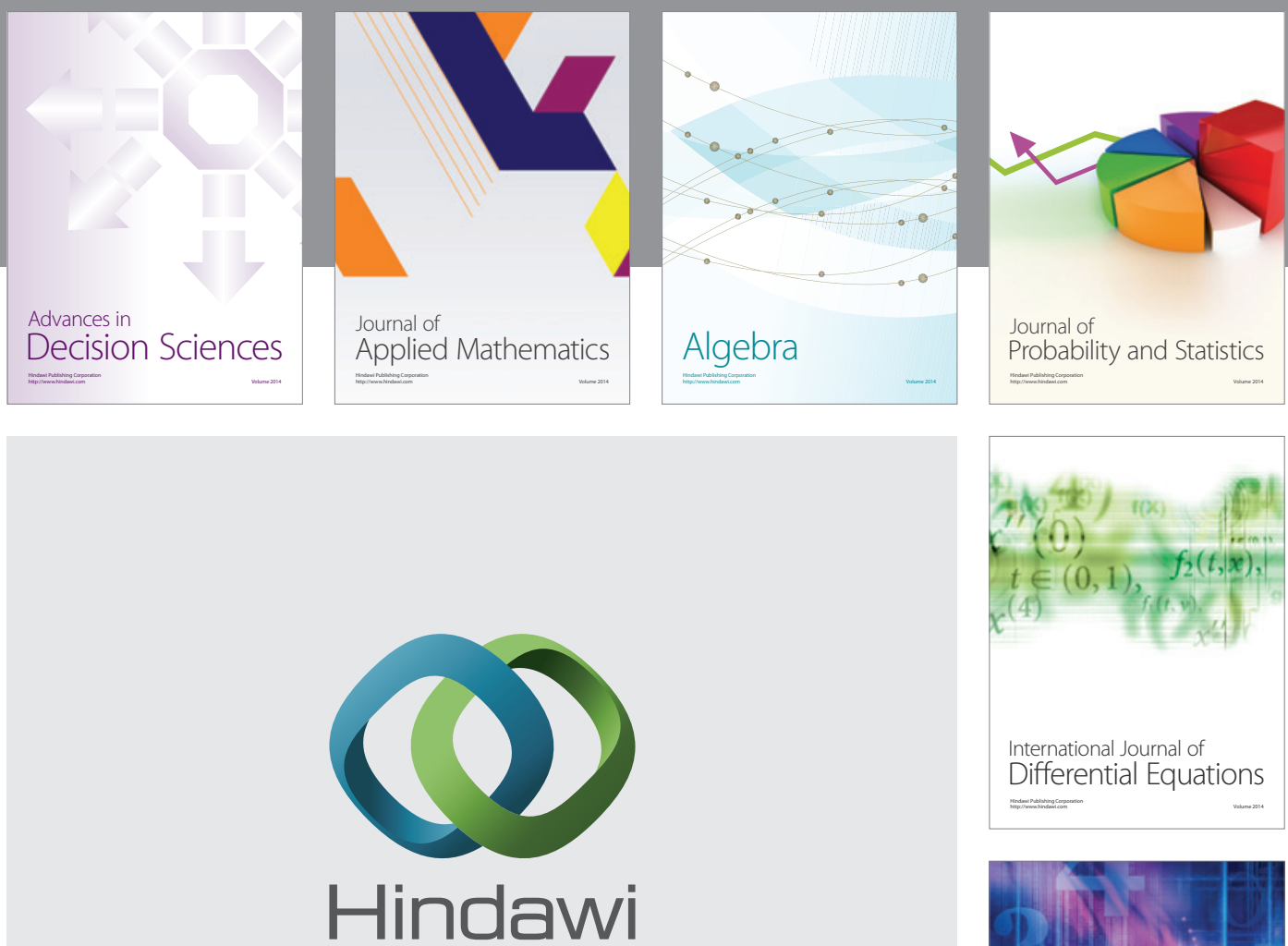

Submit your manuscripts at http://www.hindawi.com
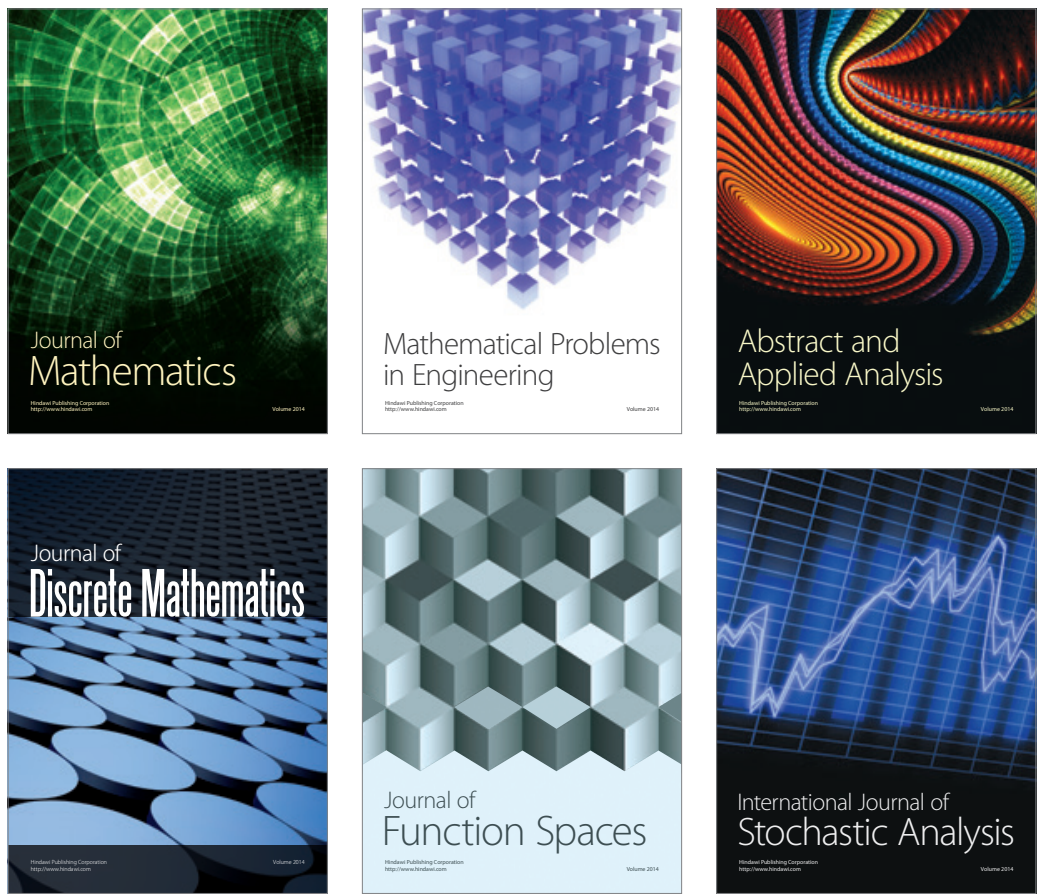

Journal of

Function Spaces

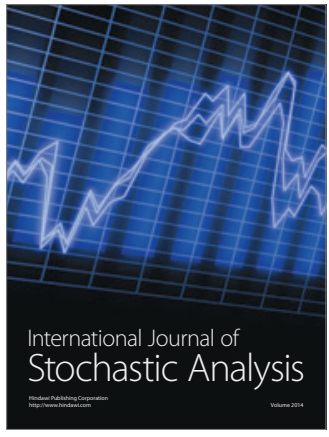

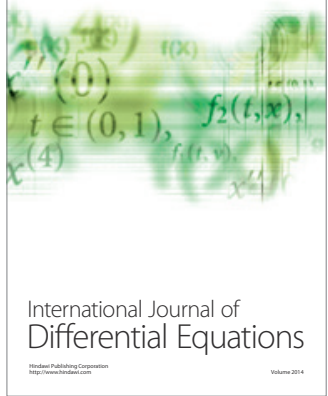
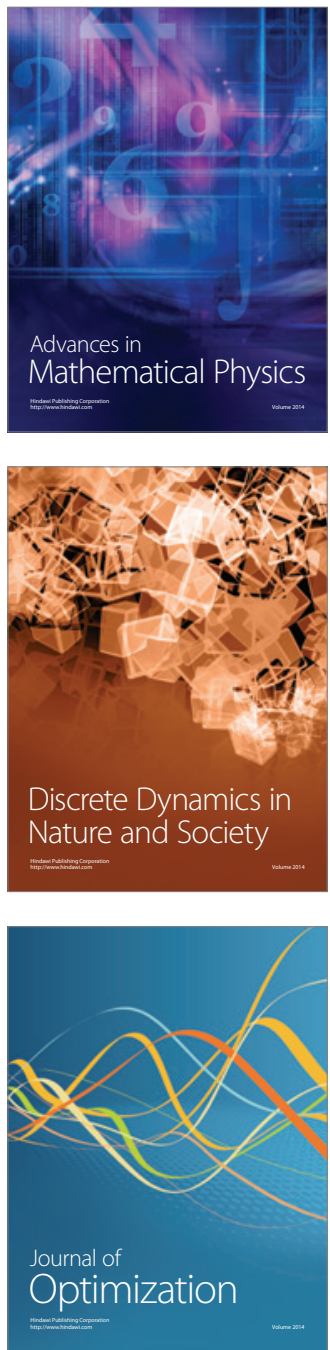